\title{
Neutrophil extracellular traps promote liver micrometastasis in pancreatic ductal adenocarcinoma via the activation of cancer-associated fibroblasts
}

\author{
SHIN TAKESUE ${ }^{1}$, KENOKI OHUCHIDA ${ }^{1}$, TOMOHIKO SHINKAWA ${ }^{1}$, YOSHIKI OTSUBO ${ }^{1}$, \\ SOKICHI MATSUMOTO ${ }^{1}$, AKIKO SAGARA ${ }^{1}$, AKIKO YONENAGA ${ }^{1}$, YOHEI ANDO ${ }^{1}$, SHIN KIBE ${ }^{1}$, \\ HIROMICHI NAKAYAMA ${ }^{1}$, CHIKA IWAMOTO $^{2}$, KOJI SHINDO ${ }^{1}$, TAIKI MORIYAMA ${ }^{3}$, \\ KOHEI NAKATA ${ }^{1}$, YOSHIHIRO MIYASAKA ${ }^{1}$, TAKAO OHTSUKA ${ }^{1}$, HIROKI TOMA ${ }^{1}$, \\ YOHEI TOMINAGA ${ }^{1}$, KAZUHIRO MIZUMOTO ${ }^{1}$, MAKOTO HASHIZUME ${ }^{2}$ and MASAFUMI NAKAMURA ${ }^{1}$ \\ Departments of ${ }^{1}$ Surgery and Oncology, ${ }^{2}$ Advanced Medical Initiatives, and ${ }^{3}$ Endoscopic Diagnostics and \\ Therapeutics, Graduate School of Medical Sciences, Kyushu University, Fukuoka 812-8582, Japan
}

Received December 17, 2018; Accepted November 18, 2019

DOI: $10.3892 /$ ijo.2019.4951

\begin{abstract}
Cancer-associated fibroblasts (CAFs) promote the progression of pancreatic ductal adenocarcinoma (PDAC) via tumor-stromal interactions. Neutrophil extracellular traps (NETs) are extracellular DNA meshworks released from neutrophils together with proteolytic enzymes against foreign pathogens. Emerging studies suggest their contribution to liver metastasis in several types of cancer. Herein, in order to investigate the role of NETs in liver metastasis in PDAC, the effects of NET inhibitors on spontaneous PDAC mouse models were evaluated. It was demonstrated that DNase I, a NET inhibitor, suppressed liver metastasis. For further investigation, further attention was paid to liver micrometastasis and an experimental liver metastasis mouse model was used that was generated by intrasplenic tumor injection. Furthermore, DNase I also suppressed liver micrometastasis and notably, CAFs accumulated in metastatic foci were significantly decreased in number. In vitro experiments revealed that pancreatic cancer cells induced NET formation and consequently NETs enhanced the migration of hepatic stellate cells, which was the possible origin of CAFs in liver metastasis. On the whole, these results suggest that NETs promote liver micrometastasis in PDAC via the activation of CAFs.
\end{abstract}

Correspondence to: Dr Kenoki Ohuchida or Dr Masafumi Nakamura, Department of Surgery and Oncology, Graduate School of Medical Sciences, Kyushu University, 3-1-1 Maidashi, Fukuoka 812-8582, Japan E-mail: kenoki@surg1.med.kyushu-u.ac.jp

E-mail:mnaka@surg1.med.kyushu-u.ac.jp

Key words: neutrophil extracellular traps, liver metastasis, pancreatic ductal adenocarcinoma

\section{Introduction}

Pancreatic ductal adenocarcinoma (PDAC) is the fourth leading cause of cancer-related mortality in western countries and has a 5 -year survival rate of $<8 \%$ (1). This dismal outcome partly results from its aggressive metastatic nature. In addition, up to $80 \%$ of patients with PDAC already have distant organ metastasis at the time of diagnosis and they rarely receive survival benefits from surgical resection. Furthermore, the lack of effective therapeutics for the treatment of metastasis makes it difficult to overcome metastatic PDAC. Therefore, novel strategies with which to prevent metastasis are urgently required in order to improve the outcome of patients with pancreatic cancer.

PDAC is characterized by abundant stroma known as 'desmoplasia', not only in primary tumors, but also in liver metastasis. Desmoplasia constitutes up to $90 \%$ of the total tumor volume and contains extracellular matrix (ECM) and stromal cells, such as immune cells, vascular endothelial cells and cancer-associated fibroblasts (CAFs). CAFs are the major component of the tumor microenvironment, as well as metastatic sites. In addition, emerging studies have revealed that CAFs contribute to tumor growth, invasion and metastasis via the secretion of various growth factors, cytokines, chemokines and the production of ECM proteins (2-5).

Neutrophils are the most abundant cell population in leukocytes and kill microbes through a variety of mechansisms, such as through phagocytosis, the release of reactive oxygen intermediates, cytokines and lytic enzymes, which can also damage cancer cells. However, previous studies have revealed that neutrophils also have pro-tumorigenic roles; clinical investigations have indicated that high levels of neutrophils both in the tumor and in the circulation (6-9), as well as a high neutrophil-to-lymphocyte ratio, have a close association with worse oncological outcomes of patients with solid tumors (10-14). Furthermore, neutrophils accelerate tumor progression, not only in the primary tumor, but also in the metastatic tumor through various mechanism, 
such as the recruitment of immuno-suppressive regulatory $\mathrm{T}$ cells (15), angiogenesis (16) and the establishment of the pre-metastatic niche (17). On the other hand, in 2004, Brinkmann et al first documented a novel neutrophil function termed neutrophil extracellular traps (NETs) (18). NETs are extracellular networks consisting of DNA filaments released from neutrophil nuclei together with antimicrobial peptides, proteases and histones derived from neutrophil granules in response to infection. NETs have been demonstrated to capture and destroy foreign pathogens, and consequently contribute to host defense, while it has been also reported that NETs are associated with various human pathologies, not only in infectious diseases, but also in non-infectious diseases, such as preeclampsia (19), rheumatoid arthritis (20), thrombosis (21-23), and lung fibrosis (24), as well as cancer metastasis $(25,26)$. However, the mechanisms through which NETs promote cancer metastasis and their role in PDAC progression remain largely unknown.

The present study demonstrated the role of NETs in liver metastasis from PDAC, with particular focus on micrometastasis. DNase I, a NET inhibitor, was found to suppress liver metastasis in both spontaneous and experimental liver metastasis mouse models. Neutrophils were recruited during the very early stages of liver micrometastasis followed by CAF activation, which was attenuated by DNase I. The findings of the present study suggest that NETs promote liver metastasis, and indicate that they may thus be a promising therapeutic target which can be used to suppress the liver metastasis of PDAC.

\section{Materials and methods}

Human samples. The collection of human PDAC tumor samples was approved by the Ethics Committee of Kyushu University. Written informed consent was obtained from the patients as required. Two PDAC samples from a 65-year-old and 72-year-old male who underwent pancreatoduodenectomy in 2014 at Kyushu University were collected.

Mouse strains. The following strains were used: Kras ${ }^{\mathrm{LSL}-\mathrm{G} 12 \mathrm{D}}$ PDX1-Cre and $\operatorname{Trp5} 3^{\mathrm{R} 172 \mathrm{H}}$ (KPC mouse); Kras ${ }^{\text {LSL-G12D }}$, PDX1-Cre, Trp53 ${ }^{\mathrm{R} 172 \mathrm{H}}$ and Luciferase ${ }^{\mathrm{LSL}}$ (KPCL mouse); and C57BL/6 mice. All mice were purchased from Charles River Laboratories at the age of 4 weeks and kept in a P2 type mouse room with a temperature of $20-26^{\circ} \mathrm{C}$ and a humidity of $40-60 \%$ and were provided with free access to food and water. The KPC mice and KPCL mice were used as spontaneous PDAC mouse models. Luminescence in the upper abdomen of the KPCL mice was observed using an IVIS image system (Caliper Life Sciences) following an intraperitoneal injection of $150 \mathrm{mg} /$ body D-Luciferin potassium salt (\#LK10000; OZ Biosciences). The KPCL mice were sacrificed by cervical dislocation under anesthesia with isoflurane (5\% for induction and $2 \%$ for maintenance) when they lost $>10 \%$ of their body weight or the tumors reached a size of $2 \mathrm{~cm}$ in the largest diameter and death verification were conducted by confirming the lack of heartbeat, respiration and pupillary response to light. All mouse experiments were approved by the Ethics Committee of Kyushu University and all mice were used in accordance with institutional guidelines.
Cells and culture conditions. Murine pancreatic cancer cells (PCCs) were established from PDAC tissues obtained from KPC mice using the outgrowth method, as previously reported (27), and cultured in DMEM supplemented with $10 \%$ fetal bovine serum (FBS). Cells were confirmed to be PCCs by morphology and immunofluorescent staining for cytokeratin 19 (data not shown). The PCC1, PCC-Li1, 2 and PCC-Lu1 cells were established from primary, liver metastasis and lung metastasis, respectively. All of the established murine cancer cell lines were not authenticated. Human hepatic stellate cells (HSCs) were purchased from KAC (\#BBHPFS-01) and cultured in DMEM supplemented with $10 \%$ FBS. Although immortalized HSCs, such as LX-1 and LX-2 are used in several experiments, this study used HSCs which are not immortalized to avoid the effects of immortalization on their biological features. In addition, the authors also tried to isolate primary HSCs from murine liver by the widely used in vivo digestion method as previously described (28). However, the isolated HSCs were no suitable for use in the experiments due to the low yield and low viability.

Visualization of extracellular DNA in vivo. In vivo extracellular DNA released along with NET formation was labeled with $5 \mu \mathrm{M}$ SYTOX Green (Thermo Fisher Scientific) membrane impermeable nucleic acid stain. SYTOX Green was injected intravenously $10 \mathrm{~min}$ before harvesting tumors from tumor-bearing KPCL mice. Each explanted tumor was cut into two sections and the cross-sections and sliced sections were observed under a fluorescence microscope (BZ-9000; Keyence).

Experimental liver micrometastasis model. Green fluorescent protein (GFP)-transfected murine PCCs were generated using lentivirus according to the manufacturer's instructions. Blasticidine S hydrochloride (\#15205; Sigma-Aldrich) was used for selection for $>3$ weeks. A total of $1 \times 10^{5}$ cells was injected into the spleens of immunocompetent C57BL/6 mice. Mice were sacrificed by cervical dislocation under anesthesia with isoflurane (5\% for induction and $2 \%$ for maintenance) at days 1-4 after the tumor injection.

DNase I treatment in mouse models. In both spontaneous and experimental liver metastasis mouse models, $75 \mathrm{U}$ of DNase I was injected daily into the peritoneal cavity. In the experimental liver metastasis mouse model, DNase I administration was commenced $10 \mathrm{~min}$ prior to the intrasplenic tumor injection.

Murine neutrophil isolation. Murine neutrophils were isolated from the bone marrow of femurs and tibias of C57BL/6 mice, as described previously (29). In brief, harvested bone marrow cells suspended in Hank's buffered salt solution (HBSS) without $\mathrm{Ca}^{2+} / \mathrm{Mg}^{2+}$ were centrifuged at $1,000 \mathrm{x}$ for $5 \mathrm{~min}$ at $4^{\circ} \mathrm{C}$ and the pellets were resuspended in red blood cell lysing buffer (BD Pharm Lyse, BD Biosciences). After washing with HBSS, neutrophils were separated from mononuclear cells by layering the cell suspension on $81 \%$ Percoll (GE Healthcare) and under $62 \%$ Percoll following centrifugation at $1,000 \mathrm{x} \mathrm{g}$ for $20 \mathrm{~min}$ at $4^{\circ} \mathrm{C}$. The neutrophils were then collected from the 
enriched layer between the 81 and $62 \%$ Percoll and cultured in RPMI-1640 medium supplemented with $10 \%$ FBS. The purity of the isolated neutrophils was determined by flow cytometry (SH800; SONY) using PE-conjugated anti-Ly6G antibody (\#127607; BioLegend) and FITC-conjugated anti-CD11b antibody (\#101205; BioLegend) and NETs were induced by the addition of $40 \mathrm{nM}$ phorbol-12-myristate 13-acetate (PMA; Sigma-Aldrich).

Immunohistochemistry. In vivo tumor tissues were immunostained as previously described (30) with the following primary antibodies: Rabbit anti-GFP antibody (1:500, \#598; MBL), mouse anti- $\alpha$-smooth muscle actin ( $\alpha$-SMA) antibody (1:100, \#M0851; Dako), rabbit anti-F4/80 antibody (1:100, \#ab234530; Abcam), rat anti-Ly6G antibody (1:100, \#551459; BD Pharmingen; BD Biosciences), goat anti-myeloperoxidase antibody (MPO; $15 \mu \mathrm{g} / \mathrm{ml}, \mathrm{AF} 3667$; R\&D Systems) and rabbit anti-citrullinated-histone $\mathrm{H} 3$ antibody (Cit-H3) (1:100, ab5103; Abcam) and the following secondary antibodies: EnVisionp System-HRP Labeled Polymer Anti-Rabbit (\#K4003; Dako), EnVisionp System-HRP Labeled Polymer Anti-Mouse (\#K4001; Dako), goat anti-rat IgG antibody (1:500, \#ab97057; Abcam). Tissues were incubated with the primary antibodies overnight at $4^{\circ} \mathrm{C}$ and stained with the secondary antibodies.

Immunofluorescence staining. Immunofluorescence staining was performed as previously described (31) with the following antibodies: Rabbit anti-GFP antibody (1:500, \#598; MBL), mouse anti- $\alpha$-SMA antibody (1:100, M0851; Dako), rat PE-conjugated anti-Ly6G antibody (1:50,\#127607; BioLegend), rabbit anti-Cit-H3 antibody (1:100, ab5103; Abcam), Alexa 546-conjugated anti mouse IgG antibody (1:200, \#A-11030; Invitrogen; Thermo Fisher Scientific) and Alexa 488-conjugated anti-rabbit IgG antibody (1:200, \#A-11034; Invitrogen; Thermo Fisher Scientific). Slides were visualized using a fluorescence microscope (BZ-9000; Keyence).

Quantification of NETs induced by PCCs. Freshly isolated neutrophils from C57BL/6 mice were seeded in the lower wells of 24-well Transwell chambers at a density of $2.0 \times 10^{4} /$ well in $750 \mu \mathrm{l}$ of RPMI supplemented with $10 \% \mathrm{FBS}$. For indirect co-culture, murine PCCs $\left(4.0 \times 10^{4} /\right.$ well), derived from either primary tumor or metastatic tumor, in $250 \mu \mathrm{l}$ of RPMI supplemented with $10 \%$ FBS, were seeded in the upper well with $8 \mu \mathrm{m}$ pores. For cancer supernatant treatment, the murine PCCs were cultured in RPMI without FBS for $72 \mathrm{~h}$ and the cells in the supernatants were removed using Millex filters $(0.22 \mu \mathrm{m}$ pore size; Merck) and were then concentrated with centrifugal filter tubes. Collected supernatants were added to fresh RPMI and adjusted to the same concentration as the original medium. Following $24 \mathrm{~h}$ of either indirect co-culture or supernatant treatment, neutrophils in the lower wells were fixed with 4\% paraformaldehyde and incubated with rabbit anti-Cit-H3 antibody (1:50, ab5103; Abcam) for $2 \mathrm{~h}$ at room temperature followed by $1 \mathrm{~h}$ of incubation with FITC-conjugated secondary antibody (1:200, ab6717; Abcam) and DAPI at room temperture. Neutrophils that produced NETs were double-stained with FITC and DAPI and observed under a fluorescence micro- scope. The percentage of neutrophils that released NETs was measured.

Migration assay. The HSC migratory capabilities were investigated by counting the number of migrating HSCs through $8-\mu \mathrm{m}$ pores of Transwell chamber membranes (BD Biosciences), as previously described (30). For co-cultures with or without PCCs (2.0x10 $/$ well), neutrophils $\left(2.0 \times 10^{5} /\right.$ well $)$ and apocynin $(10 \mu \mathrm{M})$ in $750 \mu \mathrm{l}$ of DMEM supplemented with $10 \%$ FBS in the lower chambers of 24-well dishes, HSCs were seeded with or without apocynin in the upper chambers and incubated at $37^{\circ} \mathrm{C}$ for $24 \mathrm{~h}$. Following incubation, cell migration was evaluated by counting the number of cells that migrated through the Transwell chambers. Migrated cells on the bottom surface of the membranes were fixed with $70 \%$ ethanol and stained with hematoxylin for $5 \mathrm{~min}$ and eosin for $3 \mathrm{~min}$. Cells in 5 random fields at x200 magnification were counted under a light microscope (BZ-9000; Keyence). Each experiment was performed in triplicate.

Cell proliferation assay. Cell proliferation was evaluated using the Cell Titer-Glo luminescent cell viability assay. PCC1 and HSCs were seeded in 96-well polystyrene cell culture microplates (\#655083; Greiner Bio-One International) at a density of $1 \times 10^{3}$ cells/well, and $24 \mathrm{~h}$ after seeding, $100 \mathrm{ml}$ medium was replaced. The medium was replaced with fresh Dulbecco's modified Eagle's medium (DMEM) containing 10\% FBS with 5,10 , or $15 \mathrm{U} / \mathrm{ml}$ DNase I or $\mathrm{H}_{2} \mathrm{O}$. Cell proliferation was evaluated after $0,24,48$ and $72 \mathrm{~h}$ of culture according to the manufacturer's instructions.

Reverse transcription-quantitative polymerase chain reaction. Total RNA was extracted from the PSCs using a High Pure RNA Isolation kit (\#11828665001, Roche) according to the manufacturer's instructions. RT-qPCR) was performed using the SYBR-Green RT-PCR kit (\#170-8892; Bio-Rad Laboratories) and the CFX96 Real-Time PCR System (Bio-Rad Laboratories). The reactions were incubated at $50^{\circ} \mathrm{C}$ for $10 \mathrm{~min} ; 95^{\circ} \mathrm{C}$ for $1 \mathrm{~min} ; 95^{\circ} \mathrm{C}$ for $10 \mathrm{sec} ; 60^{\circ} \mathrm{C}$ for $30 \mathrm{sec}$ for 39 cycles; $60^{\circ} \mathrm{C}$ for $5 \mathrm{sec} ; 95^{\circ} \mathrm{C}$ for $5 \mathrm{sec}$. The results were analyzed using the $2^{-\Delta \Delta \mathrm{Cq}}$ method (32). Primers for actin alpha 2, smooth muscle (Acta2) and collagen type i alpha 1 chain (Col1A1) were purchased from Takara. Glyceraldehyde-3-phosphate dehydrogenase (GAPDH) was used to normalize messenger RNA (mRNA) expression. The primers used were as follows: mouse GAPDH forward, 5'-TGT GTCCGTCGTGGATCTGA-3' and reverse, 5'-TTGCTGTTG AAGTCGCAGGAG-3'; mouse Acta2 forward, 5'-CAGATG TGGATCAGCAAACAGGA-3' and reverse, 5'-GACTTAGAA GCATTTGCGGTGGA-3'; and mouse Col1A1 forward, 5'-GA CATGTTCAGCTTTGTGGACCTC-3' and reverse, 5'-GGG ACCCTTAGGCCATTGTGTA-3'.

Statistical analysis. Results are presented as the means \pm SD. For experiments comparing differences between 2 groups, Student's t-tests were used. For experiments comparing $>2$ groups, ANOVA followed by Tukey's or Dunnett's multiple comparisons tests were used. $\alpha$-SMA-positive areas in the micrometastases were analyzed using the Wilcoxon test. Statistical significance was defined as $\mathrm{P}<0.05$. All statistical analyses were performed using JMP 11 software (SAS Institute). 


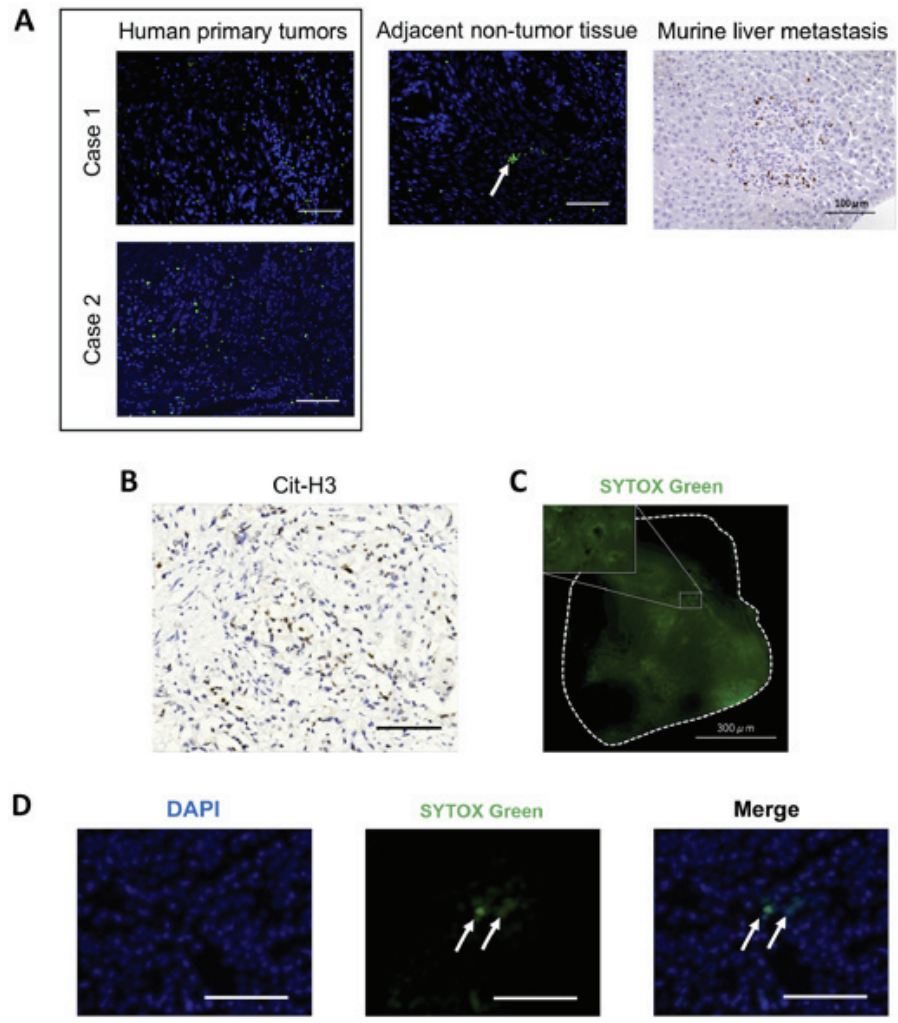

Figure 1. PDAC cells trigger neutrophil recruitment and NET formation. (A) Neutrophils infiltrating human primary PDAC, adjacent non-tumor tissue and murine liver metastatic PDAC tumors stained with Ly6G. White arrow indicates neutrophils in a blood vessel. Scale bar, $100 \mu \mathrm{m}$. (B) Representative image of NETs, positive for Cit-H3, in the liver metastasis of KPCL mouse. Scale bar, $100 \mu \mathrm{m}$. (C) Representative image of extracellular DNA in the tumor of a KPCL mouse visualized with SYTOX Green administered prior to killing. The cross-sections of the bulk tumors were observed by fluorescence microscopy. (D) Representative microscopic images of extracellular DNA stained with SYTOX Green in the sliced tumor in (C). White arrows indicate DNA stained with SYTOX Green. Scale bar, $50 \mu \mathrm{m}$. PDAC, pancreatic ductal adenocarcinoma; NETs, neutrophil extracellular traps.

\section{Results}

PCCs trigger neutrophil recruitment and NET formation in $P D A C$. To investigate the accumulation of neutrophils in PDAC tumors, specimens obtained from human primary PDAC and KPC mouse liver metastatic tumors were first analyzed using immunohistochemistry or immunofluorescence staining. In both tissues, the Ly6G-positive neutrophil density was higher in the tumors than in the adjacent non-tumor tissue. The majority of the neutrophils in the human non-tumor tissues were located in the blood vessels (Fig. 1A). To investigate NET formation in the PDAC tumors, immunohistochemistry of citrullinated-histone $\mathrm{H} 3$ was performed. Histone $\mathrm{H} 3$ is known to be citrullinated during the process of NET formation (21). Immunohistochemical analysis revealed the infiltration of citrullinated-histone H3-positive cells to the liver metastases of KPCL mice (Fig. 1B). In addition, to visualize NETs, SYTOX Green, a membrane impermeable nucleic acid stain that stains extracellular DNA released along with NET formation, was intravenously administered to KPCL mice before harvesting the tumors. Heterogeneously distributed extracellular DNA was detected during the direct observation of tumor cross-sections (Fig. 1C), and extracellular DNA was also observed in the sliced sections (Fig. 1D).

Treatment with NET inhibitor prevents liver metastasis and suppresses tumor growth in KPCL mice. NETs have previously been reported to promote the blood-borne metastasis of breast and colon cancer in an experimental liver metastasis model $(26,29,33)$; however, the significance of NETs in PDAC remains unknown. Therefore, this study investigated the effect of DNase I, which is known to be a NET inhibitor, on spontaneous PDAC tumors in KPCL mice. In these mice, the luminescent intensity of the tumor increases as the tumor grows. In a preliminary experiment, it was found that approximately half of the palpable pancreatic tumors in the KPC mice emerged at the age of 8-12 weeks and grew over time (Fig. S1). To investigate the effect of DNase I on liver metastasis formation at a very early stage, the KPCL mice were divided into 2 groups, and daily intraperitoneal DNase I administration was commenced from the age of 8 or 13 weeks, respectively (Fig. 2A). All mice were sacrificed when they lost $>10 \%$ of their body weight.

When DNase I was administered to 8-week-old mice (Early group), the DNase I group exhibited significantly lower levels of luminescence than the control group (Fig. 2B, $\mathrm{P}<0.05)$. One of the KPCL mice in the DNase I-treated group was excluded from the analysis. By contrast, when DNase I was administered to 13 -week-old mice (Delayed group), no significant difference was observed in the levels of luminescence between the DNase I and control groups. In addition, in the DNase I group, the incidence of liver metastasis was significantly suppressed (Fig. 2C, P<0.05). These data suggest that the inhibition of NET prevents liver metastasis, whereas it is insufficient for tumors that have already grown. 


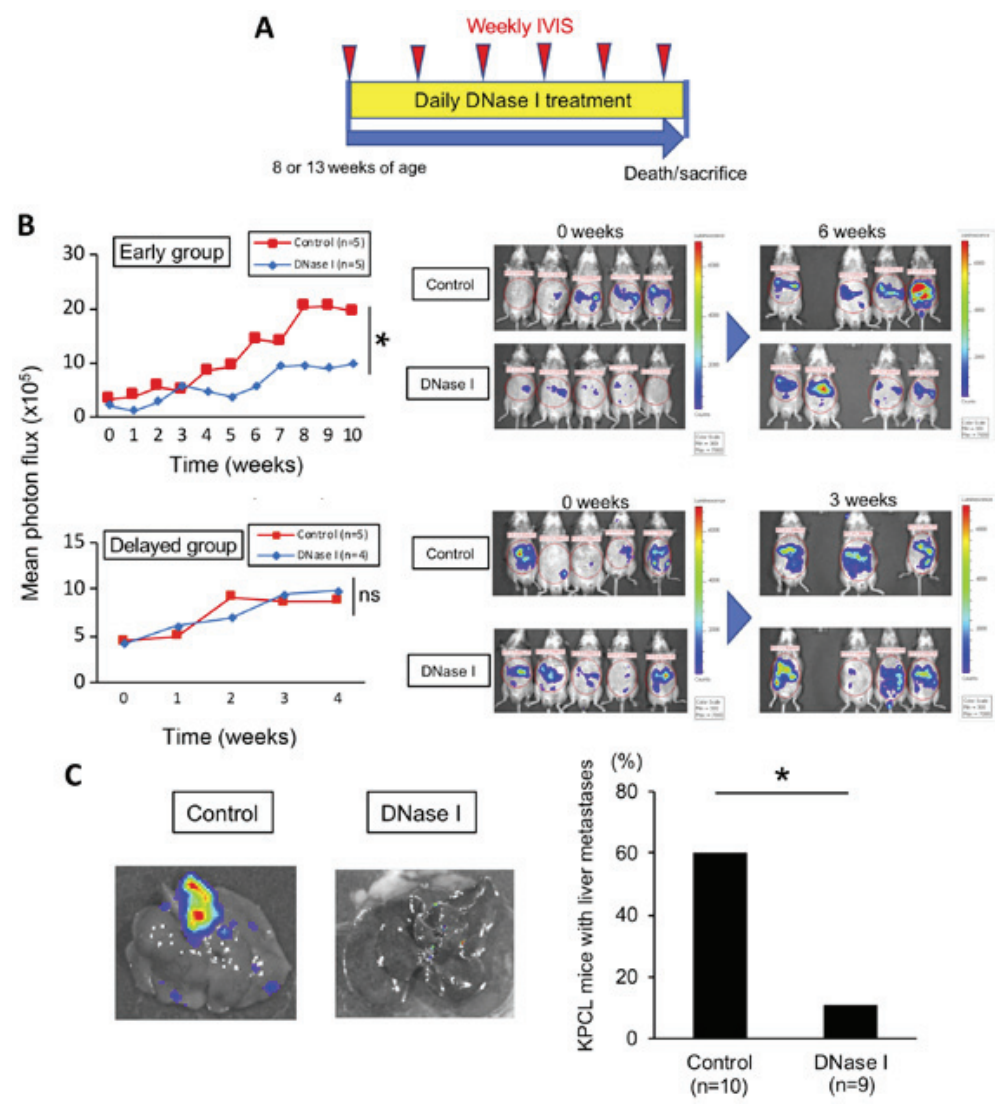

Figure 2. NET inhibitor treatment prevents liver metastasis and suppresses tumor growth in KPCL mice. (A) Schematic timeline of DNase I treatment for KPCL mice. (B) Changes in the average luminescence intensity of KPCL mice in both the Early and Delayed group (left panels) and representative IVIS images before and after DNase I treatment was commenced (right panels). (C) IVIS images of liver explant (left panels) and percentage of KPCL mice with liver metastasis (right panel). ${ }^{*} \mathrm{P}<0.05$. NET, neutrophil extracellular trap.

A
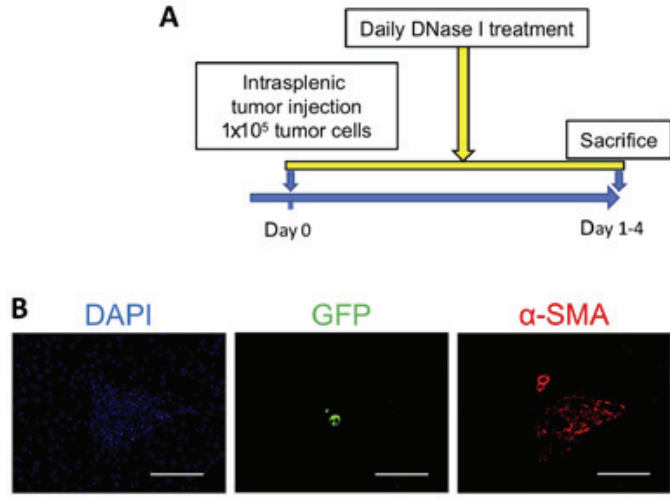

a-SMA
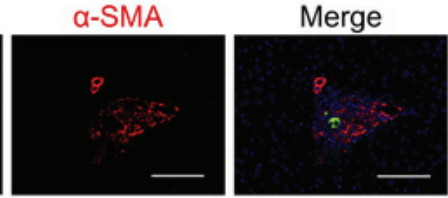

C

GFP

Ly6G

a-SMA
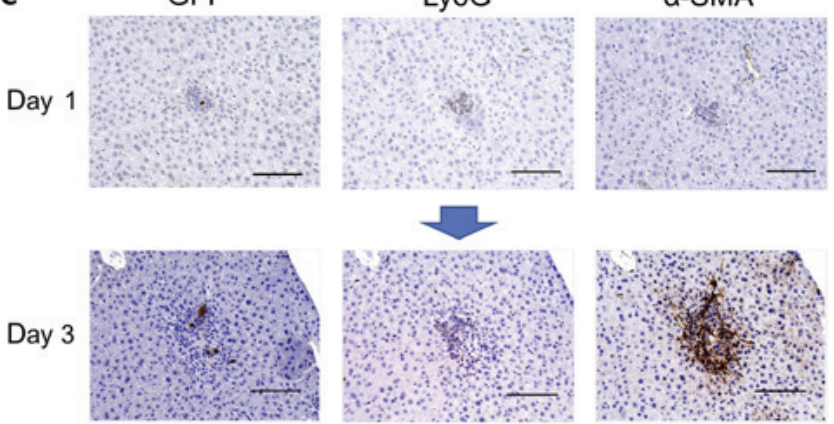

Figure 3. Neutrophil recruitment in liver metastasis precedes CAF activation. (A) Schematic timeline of experimental liver metastasis mouse model. (B) Metastatic foci with single tumor cell and surrounding CAFs in the experimental liver identified with immunofluorescent staining of GFP and $\alpha$-SMA at day 3. (C) Representative images of $\mathrm{GFP}^{+}$tumor cells, $\mathrm{Ly}_{6} \mathrm{G}^{+}$neutrophils and $\alpha$-SMA-positive CAFs in the experimental liver metastasis at days 1 and 3. Scale bars, $100 \mu \mathrm{m}$. CAF, cancer-associated fibroblast; GFP, green fluorescent protein; $\alpha$-SMA, $\alpha$-smooth muscle actin. 
A
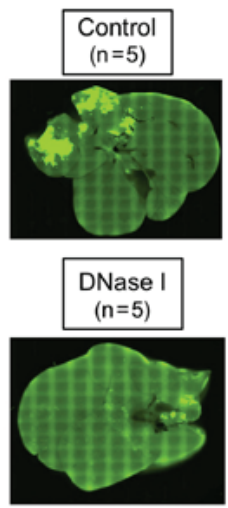

C

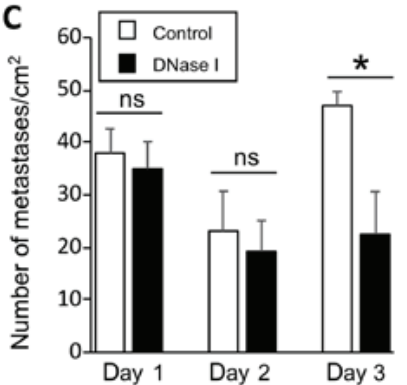

D

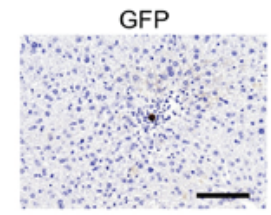

a-SMA

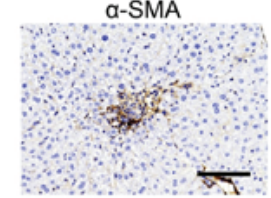

B
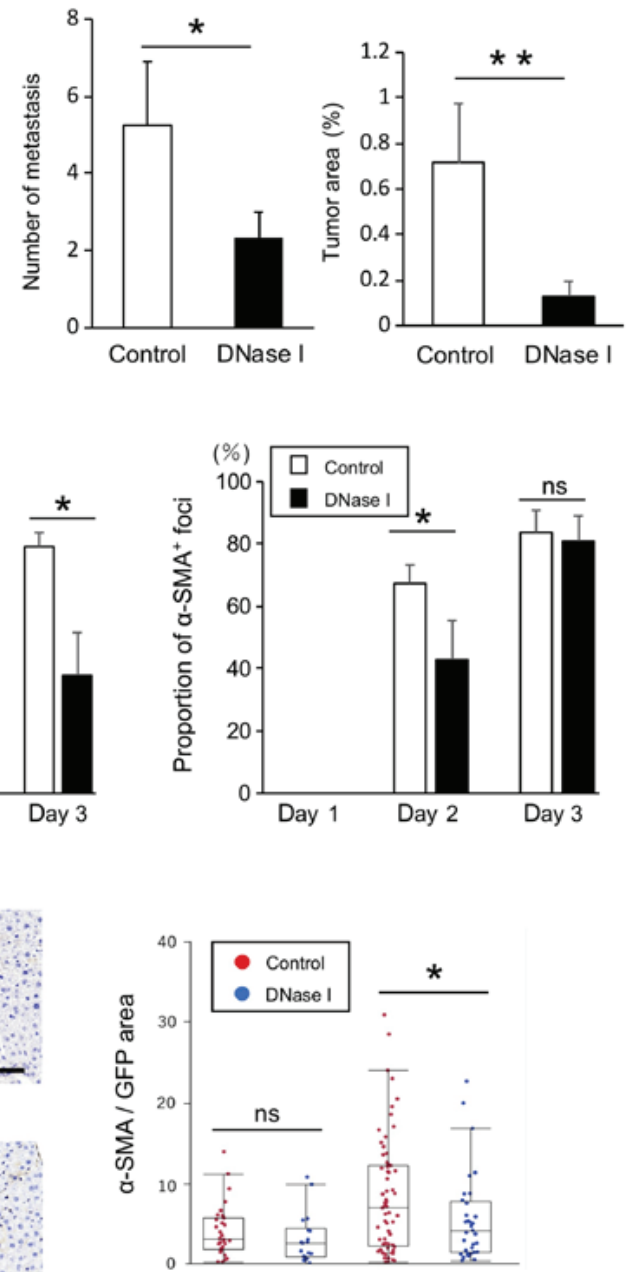

Day 2 Day 3

Figure 4. DNase I prevents liver metastasis and CAF activation in an experimental liver micrometastasis mouse model. (A) Representative images of liver explants acquired using fluorescence microscopy. (B) Quantification of the number of metastatic regions and tumor area in the control group and DNase I-treated group. Data shown represent the means \pm SD. (C) Quantification of the density of metastatic foci (left panel) and the percentage of $\alpha$-SMA-positive foci (right panel) at days 1-3 by immunohistochemical analysis of GFP and $\alpha$-SMA ( $n=3$, each group in each day.) Data shown represent the means \pm SD. (D) Representative immunohistochemical images of micrometastatic foci (left panel). $\alpha$-SMA/GFP ratio in each micrometastatic foci at days $2-3$ (right panel). Scale bar, $50 \mu \mathrm{m}$. ${ }^{*} \mathrm{P}<0.05,{ }^{* *} \mathrm{P}<0.001 ;$ ns, not significant. CAF, cancer-associated fibroblast; GFP, green fluorescent protein; $\alpha$-SMA, $\alpha$-smooth muscle actin.

Neutrophil recruitment in liver metastasis precedes $C A F$ activation. To evaluate the type of stromal cells accumulating around cancer cells during the very early stages of liver metastasis, an experimental liver micrometastasis mouse model was used, in which mice were transplanted with cancer cells by an intrasplenic injection with or without DNase I (Fig. 3A). In this model, PDAC cells from KPC mice were labeled with GFP to allow the identification of the PDAC cells even at the single cell level by the immunofluorescence staining of GFP (Fig. 3B). When examined microscopically, regardless of DNase I treatment, metastatic foci containing only a couple of GFP-positive PCCs were observed at post-injection day 1 surrounded by Ly6G-positive neutrophils, whereas $\alpha$-SMA-positive CAFs were not observed at that time. However, at post-injection day 3 , the dense accumulation of CAFs was observed around the PDAC cells, while neutrophils still existed (Fig. 3C).

Macrophages, which have also been reported to play a pro-tumorigenic role in liver metastasis $(34,35)$, were not observed during days 1-3 (Fig. S2). The time lag between neutrophils and macrophages during the recruitment indicated their differences in the mechanisms of recruitment and in their role in the initiation of metastasis.

NET inhibitor prevents liver metastasis and the recruitment of activated CAFs in an experimental liver micrometastasis mouse model. The present experimental results for the KPCL mice treated with the DNase I NET inhibitor suggested that NETs were associated with tumor progression, particularly at the early stage. Therefore, in order to focus on liver micrometastasis, an experimental liver metastasis model was used as described above. GFP-transfected tumor cells derived from KPC mice were injected into the spleens of immunocompetent mice, which were sacrificed from day 1 to 4 . To examine the effects of NET inhibitor on liver micrometastasis formation, DNase I was administered daily to this mouse model. First, the number of metastatic nodules in DNase I-treated and control 
A

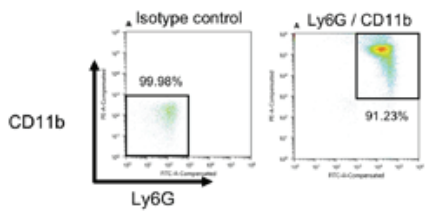

C

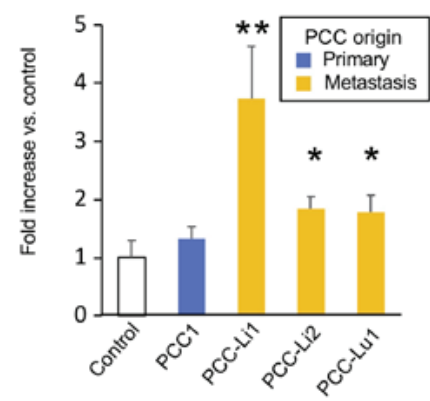

B
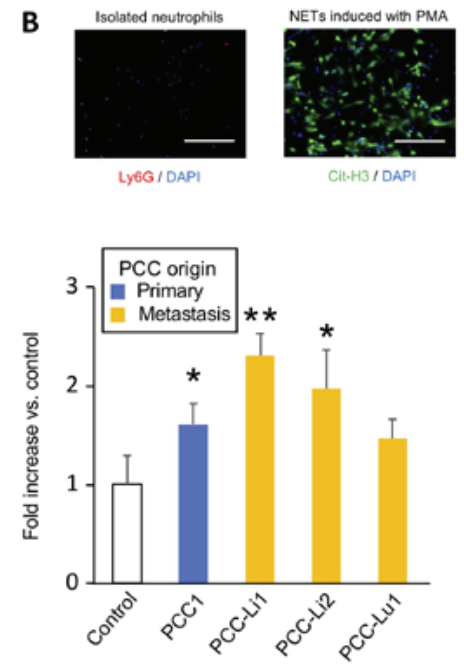

PCC-Li1

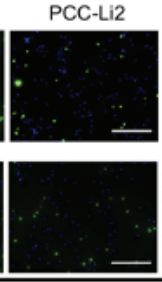

PCC-Li2

PCC-Lu1
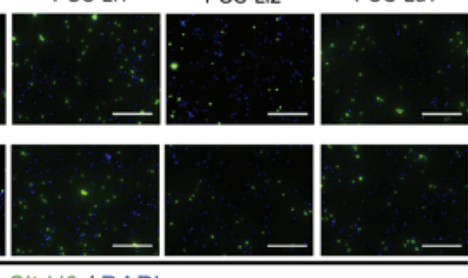

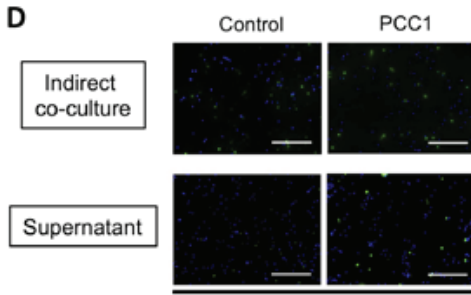

Cit-H3 / DAP|

Figure 5. PCCs induce NET formation in vitro. (A) Purity of isolated neutrophils was measured using flow cytometry with Ly6G and CD11b. (B) Isolated neutrophils (left panel, red) and induced NETs with $40 \mathrm{nM}$ PMA (right panel, green). Scale bar, $100 \mu \mathrm{m}$. (C) Quantification of NETs induced by murine PCCs. The blue and yellow columns indicate the NETs induced by PCCs established from primary and metastatic tumors, respectively. Data shown represent the means \pm SD. (D) Representative images of induced NETs by PCCs. Neutrophils that released NETs were visualized by immunofluorescent staining of Cit-H3. Scale bars, $100 \mu \mathrm{m} .{ }^{*} \mathrm{P}<0.05,{ }^{* *} \mathrm{P}<0.001$. PCCs, murine pancreatic cancer cells; NET, neutrophil extracellular trap.

mice at day 4 was evaluated. Fluorescence microscopic analysis revealed a significant decrease in both the number and area of metastatic nodules in the DNase I-treated group compared with the control group (Fig. 4A and B). Furthermore, immunohistochemical analysis of GFP revealed that there was no significant difference in the number of metastatic nodules between the 2 groups at days 1 and 2, while the DNase-I-treated mice exhibited significantly fewer liver micrometastasis at day 3 than the control mice ( $\mathrm{P}<0.05$; Fig. $4 \mathrm{C}$, left panel).

A recent study demonstrated that NETs promote the differentiation and function of lung fibroblasts triggered by fibrosis-related agents (24). Therefore, this study investigated the effects of DNase I on the recruitment of activated CAFs, which play a crucial role in the fibrotic stroma, in liver metastasis. The immunohistochemical analysis of experimental liver metastasis revealed that $\alpha$-SMA-positive activated CAFs were recruited to the metastatic foci from day 2 and the proportion of metastatic foci with CAFs increased over time. The proportion of metastatic foci with CAFs was significantly lower in the DNase I-treated group than the control group at day 2, while there was no difference at day 3 (Fig. 4C, right panel), as the majority of the metastatic foci were accompanied by CAFs in both groups. Furthermore, the ratio of the $\alpha$-SMA-positive area to the GFP-positive area in each metastatic focus was significantly lower in the DNase I-treated group than the control group (Fig. 4D). To examine the direct effects of DNase I on the function of PCCs and CAFs, PCCs and CAFs were treated with DNase I in vitro. By cell proliferation assay, it was confirmed that DNase I had no effect on the proliferation of PCCs and HSCs (Fig. S3). These results suggest that DNase I inhibits the recruitment of activated CAFs during the formation of metastasis.

PCCs induce NET formation in vitro. To investigate the effects of PCCs on NETs, murine neutrophils were isolated from bone marrow as previously described (29), and the purity was measured by flow cytometry with FITC-conjugated anti-Ly6G and PE-conjugated anti-CD11b (Fig. 5A). Isolated neutrophils released NETs following the addition of $40 \mathrm{nM}$ PMA (Fig. 5B). A total of $1 \times 10^{6}$ isolated murine neutrophils were indirectly co-cultured with PCCs or treated with the supernatant of PCCs for $24 \mathrm{~h}$. All cultures of PCCs derived from primary tumors or metastatic tumors induced NETs (Fig. 5C). Notably, several cultures of PCCs derived from liver and lung metastases more potently induced NETs than those from primary tumors (Fig. 5C and D).

NETs promote the activation of HSCs. The findings of the present study suggested that PCCs promote NET formation. The effects of NETs on CAF activation in vitro were then examined. A previous study demonstrated that CAFs in liver metastasis originated from resident hepatic stellate cells (HSCs) (34). Thus, this study used human hepatic stellate cells in the subsequent experiments. The mechansism through which NETs affect the migration of HSCs were examined 
A

B

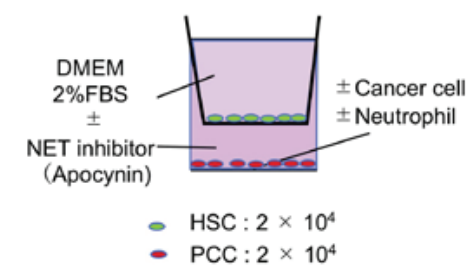

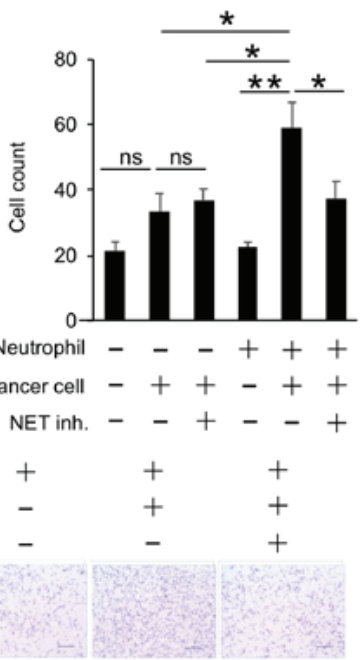

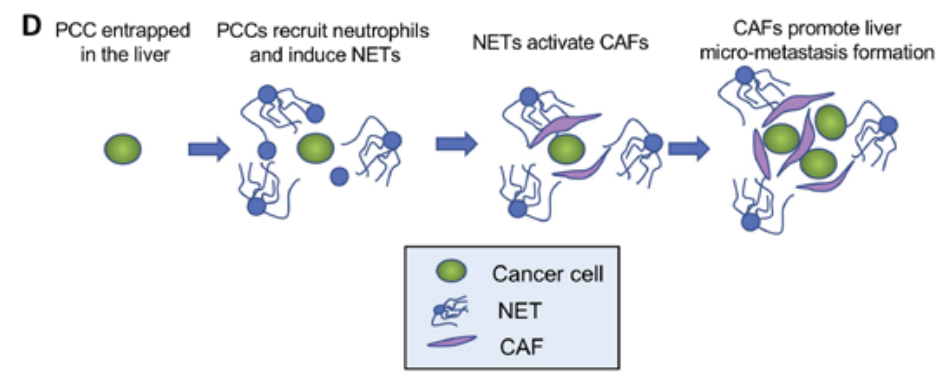

Figure 6. NETs promote the activation of HSCs. (A) Schematic representation of Transwell migration assay. (B) Quantification of migrated cell numbers under each condition. Data shown represent the mean \pm SD. (C) Representative images of migrated cells. Scale bar, $50 \mu \mathrm{m}$. (D) Schematic model showing the role of NETs in liver micrometastasis formation. ${ }^{*} \mathrm{P}<0.05,{ }^{* *} \mathrm{P}<0.001$; ns, not significant. NETs, neutrophil extracellular traps; HSCs, human hepatic stellate cells.

using Transwell migration assays (Fig. 6A). When HSCs were indirectly co-cultured with either PCCs or neutrophils alone, the number of migrated HSCs was not altered. On the contrary, when co-cultured with both PCCs and neutrophils, the number of migrated HSCs was significantly increased compared with those when HSCs were co-cultured with either PCCs or neutrophils alone. When treated with apocynin, a NET inhibitor, the increase in the number of migrated HSCs co-cultured with both PCCs and neutrophils was attenuated to the level observed with co-culture with either PCCs or neutrophils alone (Fig. 6B and C). These results suggest that PCC-enhanced NETs promote the migration of HSCs. However, quantitative PCR (qPCR) revealed that NETs had no significant effect on the expression levels of Acta2 and Col1A1 in HSCs (Fig. S4).

\section{Discussion}

Although it has been documented that DNase I suppresses blood-borne metastasis in mouse models of breast, lung and liver cancer (36-38), the underlying mechanisms have remained unclear for decades. Recent studies have revealed that DNase I suppresses metastasis via NET inhibition $(26,29)$, which leads to fewer cancer cells being entrapped in the vasculature of metastatic organs $(33,38,39)$. However, the present study, using an experimental PDAC liver metastasis model, demonstrated no difference in the number of metastatic foci between the DNase I-treated and control groups until day 2 after the injection of cancer cells, suggesting that there are other mechanisms through which DNase I can suppress tumor colonization, apart from tumor cell entrapment.

The tumor microenvironment is widely recognized to promote PDAC progression through tumor-stromal interactions. However, the involvement of tumor-stromal interactions in NET-enhanced liver metastasis is unknown. Therefore, this study examined the effect of NETs on CAFs, which play a pivotal role in the tumor-supportive microenvironment and found that NET inhibitors suppressed the recruitment of activated CAFs in the micrometastatic foci. Furthermore, the present in vitro data demonstrated that NETs enhanced the migration of HSCs, which are known to be the origin of CAFs in liver metastasis. Previous studies have demonstrated the tumor-promoting role of CAFs in metastatic colonization $(40,41)$ as well as in the primary tumor. Together with these findings, the findings of the present study suggest that NETs induce liver metastasis via the enhanced recruitment of activated CAFs.

The vast majority of previous studies on liver metastasis have mainly focused on macrometastasis $(42,43)$, resulting in a lack of concern about micrometastasis, even though it is one of the most critical steps of the metastatic cascade. This study analyzed micrometastasis in an experimental liver metastasis model. Notably, neutrophils infiltrated the liver metastatic foci within a day of intrasplenic injection of PCCs, while CAFs were absent until day 2, suggesting that neutrophils play an important role in the initial microenvironmental response before the activation of CAFs. DNase I treatment inhibited liver metastasis in this model, as well as in KPCL mice. In addition, 
immunohistochemical analysis of GFP and $\alpha$-SMA revealed that DNase I suppressed CAF activation in micrometastatic foci. Further in vitro analysis demonstrated that NETs induced by PCCs promoted the migration of HSCs, which was attenuated by NET inhibitors. By contrast, the expression levels of fibrosis-related genes in HSCs were not affected by NETs at the mRNA level. This may result from the high basal expression of these genes in HSCs related to culture activation as previously reported (28). In summary, these findings described above indicate that there NETs play a crucial role in the initial step of the liver micrometastasis formation of PDAC and that NETs can enhance micrometastasis formation through the activation of CAFs (Fig. 6D).

Conventional anticancer therapies that predominantly target cancer cells have only a limited effect on patient outcome. Even though CAFs are also assumed to be a promising therapeutic target because of their tumor-promoting nature through tumor-stroma interactions, there is no agent targeting CAFs that has a clinical application. The present data suggest that NETs are one of the candidate therapeutic targets which may be used to inhibit liver metastasis enhanced via CAF activation in PDAC.

\section{Acknowledgements}

The authors would like to thank Ms. E. Manabe, Mrs. S. Sadatomi (Department of Surgery and Oncology, Kyushu University Hospital), and members of the Research Support Center. The authors would also like to thank Mr. H. Nikki March, from Edanz Group (www.edanzediting. com/ac) for editing a draft of this manuscript.

\section{Funding}

This study was supported by JSPS KAKENHI (grant nos. JP17H04284, JP17K19602, JP16H05418, JP17K19605, and JP16K10601) and The Shinnihon Foundation of Advanced Medical Treatment Research.

\section{Availability of data and materials}

The datasets used during the current study are available from the corresponding author on reasonable request.

\section{Authors' contributions}

ST, KO and MN were involved in the conception and design of the study. ST, TS, YO, SM, YM, HT and YT were involved in the development of the methodology. ST, AS, AY, YA, SK, TO and $\mathrm{MH}$ were involved in the acquisition of the data. ST, KO, $\mathrm{HN}, \mathrm{CI}, \mathrm{KS}, \mathrm{TM}, \mathrm{KN}$ and $\mathrm{KM}$ were involved in the analysis and interpretation of the data. ST, KO and MN were involved in the writing, reviewing and revising of the manuscript. All authors have read and approved the final manuscript.

\section{Ethics approval and consent to participate}

This study was approved by the Ethics Committee of Kyushu University and written informed consent was obtained from the patients prior to sample collection. All mouse experiments, including the preliminary experiment were approved by the Ethics Committee of Kyushu University and all mice were used in accordance with institutional guidelines.

\section{Patient consent for publication}

Not applicable.

\section{Competing interests}

The authors of this manuscript have no competing interests.

\section{References}

1. Siegel RL, Miller KD and Jemal A: Cancer statistics, 2016. CA Cancer J Clin 66: 7-30, 2016.

2. McAllister SS and Weinberg RA: The tumour-induced systemic environment as a critical regulator of cancer progression and metastasis. Nat Cell Biol 16: 717-727, 2014.

3. Malanchi I, Santamaria-Martínez A, Susanto E, Peng H, Lehr HA, Delaloye JF and Huelsken J: Interactions between cancer stem cells and their niche govern metastatic colonization. Nature 481: 85-89, 2011.

4. Quail DF and Joyce JA: Microenvironmental regulation of tumor progression and metastasis. Nat Med 19: 1423-1437, 2013.

5. Erler JT, Bennewith KL, Cox TR, Lang G, Bird D, Koong A, Le QT and Giaccia AJ: Hypoxia-induced lysyl oxidase is a critical mediator of bone marrow cell recruitment to form the premetastatic niche. Cancer Cell 15: 35-44, 2009.

6. Schmidt H, Bastholt L, Geertsen P, Christensen IJ, Larsen S, Gehl J and von der Maase H: Elevated neutrophil and monocyte counts in peripheral blood are associated with poor survival in patients with metastatic melanoma: A prognostic model. Br J Cancer 93: 273-278, 2005.

7. Tibaldi C, Vasile E, Bernardini I, Orlandini C, Andreuccetti M and Falcone A: Baseline elevated leukocyte count in peripheral blood is associated with poor survival in patients with advanced non-small cell lung cancer: A prognostic model. J Cancer Res Clin Oncol 134: 1143-1149, 2008.

8. Schmidt H, Suciu S, Punt CJA, Gore M, Kruit W, Patel P, Lienard D, von der Maase H, Eggermont AM and Keilholz U; American Joint Committee on Cancer Stage IV Melanoma; EORTC 18951: Pretreatment levels of peripheral neutrophils and leukocytes as independent predictors of overall survival in patients with American Joint Committee on Cancer Stage IV Melanoma: Results of the EORTC 18951 Biochemotherapy Trial. J Clin Oncol 25: 1562-1569, 2007.

9. Teramukai S, Kitano T, Kishida Y, Kawahara M, Kubota K, Komuta K, Minato K, Mio T, Fujita Y, Yonei T, et al: Pretreatment neutrophil count as an independent prognostic factor in advanced non-small-cell lung cancer: An analysis of Japan Multinational Trial Organisation LC00-03. Eur J Cancer 45: 1950-1958, 2009.

10. Halazun KJ, Aldoori A, Malik HZ, Al-Mukhtar A, Prasad KR, Toogood GJ and Lodge JPA: Elevated preoperative neutrophil to lymphocyte ratio predicts survival following hepatic resection for colorectal liver metastases. Eur J Surg Oncol 34: 55-60, 2008.

11. Walsh SR, Cook EJ, Goulder F, Justin TA and Keeling NJ: Neutrophil-lymphocyte ratio as a prognostic factor in colorectal cancer. J Surg Oncol 91: 181-184, 2005.

12. Liu H, Liu G, Bao Q, Sun W, Bao H, Bi L, Wen W, Liu Y, Wang Z, Yin $\mathrm{X}$, et al: The baseline ratio of neutrophils to lymphocytes is associated with patient prognosis in rectal carcinoma. J Gastrointest Cancer 41: 116-120, 2010.

13. Kim IY, You SH and Kim YW: Neutrophil-lymphocyte ratio predicts pathologic tumor response and survival after preoperative chemoradiation for rectal cancer. BMC Surg 14: 94, 2014.

14. Liao W, Zhang J, Zhu Q, Qin L, Yao W, Lei B, Shi W, Yuan S, Tahir SA, Jin J, et al: Preoperative neutrophil-to-lymphocyte ratio as a new prognostic marker in hepatocellular carcinoma after curative resection. Transl Oncol 7: 248-255, 2014.

15. Mishalian I, Bayuh R, Eruslanov E, Michaeli J, Levy L, Zolotarov L, Singhal S, Albelda SM, Granot Z and Fridlender ZG: Neutrophils recruit regulatory T-cells into tumors via secretion of CCL17 - A new mechanism of impaired antitumor immunity. Int J Cancer 135: 1178-1186, 2014. 
16. Gordon-Weeks AN, Lim SY, Yuzhalin AE, Jones K, Markelc B, Kim KJ, Buzzelli JN, Fokas E, Cao Y, Smart S, et al: Neutrophils promote hepatic metastasis growth through fibroblast growth factor 2-dependent angiogenesis in mice. Hepatology 65: 1920-1935, 2017.

17. Wculek SK and Malanchi I: Neutrophils support lung colonization of metastasis-initiating breast cancer cells. Nature 528: 413-417, 2015.

18. Brinkmann V, Reichard U, Goosmann C, Fauler B, Uhlemann Y, Weiss DS, Weinrauch Y and Zychlinsky A: Neutrophil extracellular traps kill bacteria. Science 303: 1532-1535, 2004.

19. Gupta AK, Hasler P, Holzgreve W, Gebhardt S and Hahn S: Induction of neutrophil extracellular DNA lattices by placental microparticles and IL-8 and their presence in preeclampsia. Hum Immunol 66: 1146-1154, 2005.

20. Sur Chowdhury C, Giaglis S, Walker UA, Buser A, Hahn S and Hasler P: Enhanced neutrophil extracellular trap generation in rheumatoid arthritis: Analysis of underlying signal transduction pathways and potential diagnostic utility. Arthritis Res Ther 16: R122, 2014.

21. Brill A, Fuchs TA, Savchenko AS, Thomas GM, Martinod K, De Meyer SF, Bhandari AA and Wagner DD: Neutrophil extracellular traps promote deep vein thrombosis in mice. J Thromb Haemost 10: 136-144, 2012.

22. Wada H, Matsumoto T, Aota T, Yamashita Y, Suzuki K and Katayama N: Management of cancer-associated disseminated intravascular coagulation: guidance from the SSC of the ISTH: comment. J Thromb Haemost 14: 1314-1315, 2016. comment.

23. Demers M, Krause DS, Schatzberg D, Martinod K, Voorhees JR, Fuchs TA, Scadden DT and Wagner DD: Cancers predispose neutrophils to release extracellular DNA traps that contribute to cancer-associated thrombosis. Proc Natl Acad Sci USA 109: 13076-13081, 2012.

24. Chrysanthopoulou A, Mitroulis I, Apostolidou E, Arelaki S, Mikroulis D, Konstantinidis T, Sivridis E, Koffa M, Giatromanolaki A, Boumpas DT, et al: Neutrophil extracellular traps promote differentiation and function of fibroblasts. J Pathol 233: 294-307, 2014.

25. Park J, Wysocki RW, Amoozgar Z, Maiorino L, Fein MR, Jorns J, Schott AF, Kinugasa-Katayama Y, Lee Y, Won NH, et al: Cancer cells induce metastasis-supporting neutrophil extracellular DNA traps. Sci Transl Med 8: 361ra138, 2016.

26. Tohme S, Yazdani HO, Al-Khafaji AB, Chidi AP, Loughran P, Mowen K, Wang Y, Simmons RL, Huang H and Tsung A: Neutrophil extracellular traps promote the development and progression of liver metastases after surgical stress. Cancer Res 76: 1367-1380, 2016.

27. Kozono S, Ohuchida K, Eguchi D, Ikenaga N, Fujiwara K, Cui L, Mizumoto K and Tanaka M: Pirfenidone inhibits pancreatic cancer desmoplasia by regulating stellate cells. Cancer Res 73 : 2345-2356, 2013

28. Mederacke I, Dapito DH, Affò S, Uchinami H and Schwabe RF: High-yield and high-purity isolation of hepatic stellate cells from normal and fibrotic mouse livers. Nat Protoc 10: 305-315, 2015.

29. Park J, Wysocki RW, Amoozgar Z, et al:: Cancer cells induce metastasis-supporting neutrophil extracellular DNA traps. Sci Transl Med 8: 361ra138, 2016.

30. Ikenaga N, Ohuchida K, Mizumoto K, Cui L, Kayashima T, Morimatsu K, Moriyama T, Nakata K, Fujita H and Tanaka M: $\mathrm{CD} 10+$ pancreatic stellate cells enhance the progression of pancreatic cancer. Gastroenterology 139: 1041-1051, 1051 e1-1051.e8, 2010
31. Endo S, Nakata K, Ohuchida K, Takesue S, Nakayama H, Abe T, Koikawa K, Okumura T, Sada M, Horioka K, et al: Autophagy Is Required for Activation of Pancreatic Stellate Cells, Associated With Pancreatic Cancer Progression and Promotes Growth of Pancreatic Tumors in Mice. Gastroenterology 152: 1492-1506. e24, 2017.

32. Livak KJ and Schmittgen TD: Analysis of Relative Gene Expression Data Using Real-Time Quantitative PCR and the 2(-Delta Delta C(T)) Method. Methods 25: 402-408, 2001

33. Najmeh S, Cools-Lartigue J, Rayes RF, Gowing S, Vourtzoumis P, Bourdeau F, Giannias B, Berube J, Rousseau S, Ferri LE, et al: Neutrophil extracellular traps sequester circulating tumor cells via $\beta 1$-integrin mediated interactions. Int J Cancer 140: 2321-2330, 2017

34. Nielsen SR, Quaranta V,Linford A, Emeagi P, Rainer C, Santos A, Ireland L, Sakai T, Sakai K, Kim YS, et al: Macrophage-secreted granulin supports pancreatic cancer metastasis by inducing liver fibrosis. Nat Cell Biol 18: 549-560, 2016.

35. Costa-silva B, Aiello NM, Ocean AJ, Singh S, Zhang H, Thakur BK, Becker A, Hoshino A, Mark MT, Molina H, et al: Pancreatic cancer exosomes initiate pre-metastatic niche formation in the liver. Nat Cell Biol 17: 816-826, 2015.

36. Patutina O, Mironova N, Ryabchikova E, Popova N, Nikolin V, Kaledin V, Vlassov V and Zenkova M: Inhibition of metastasis development by daily administration of ultralow doses of RNase A and DNase I. Biochimie 93: 689-696, 2011.

37. Salganik RI, Martynova RP, Matienko NA and Ronichevskaya GM: Effect of deoxyribonuclease on the course of lymphatic leukaemia in AKR mice. Nature 214: 100-102, 1967.

38. Sugihara S, Yamamoto T, Tanaka H, Kambara T, Hiraoka T and Miyauchi Y: Deoxyribonuclease treatment prevents blood-borne liver metastasis of cutaneously transplanted tumour cells in mice. Br J Cancer 67: 66-70, 1993.

39. Cools-Lartigue J, Spicer J, McDonald B, Gowing S, Chow S, Giannias B, Bourdeau F, Kubes P and Ferri L: Neutrophil extracellular traps sequester circulating tumor cells and promote metastasis. J Clin Invest 123: 3446-3458, 2013.

40. O'Connell JT, Sugimoto H, Cooke VG, MacDonald BA, Mehta AI, LeBleu VS, Dewar R, Rocha RM, Brentani RR, Resnick MB, et al: VEGF-A and Tenascin-C produced by S100A4+ stromal cells are important for metastatic colonization. Proc Natl Acad Sci USA 108: 16002-16007, 2011.

41. Conti J and Thomas G: The role of tumour stroma in colorectal cancer invasion and metastasis. Cancers (Basel) 3: 2160-2168, 2011.

42. Sousa S, Brion R, Lintunen M, Kronqvist P, Sandholm J, Mönkkönen J, Kellokumpu-Lehtinen PL, Lauttia S, Tynninen O, Joensuu $\mathrm{H}$, et al: Human breast cancer cells educate macrophages toward the M2 activation status. Breast Cancer Res 17: 101, 2015.

43. Penheiter AR, Erdogan S, Murphy SJ, Hart SN, Lima JF, Rohakhtar FR, O'Brien DR, Bamlet WR, Wuertz RE, Smyrk TC, et al: Transcriptomic and Immunohistochemical Profiling of SLC6A14 in Pancreatic Ductal Adenocarcinoma. Biomed Res Int 2015: 593572, 2015. 